\title{
Impact of Stress on the Brain: Pathology, Treatment and Prevention
}

Neuropsychopharmacology Reviews (2016) 41, I-2; doi: I0.1038/npp.20 I5.306

W e are pleased to present the 2016 Neuropsychopharmacology Reviews Edition, which focuses on advances in our understanding of the impact of stress on the brain: from pathology to circuits, treatment to prevention. Emerging data from genetics, epigenetics, neural circuit and intergenerational models, animal models, and neuroimaging all suggest that stress affects the brain in a variety of dynamic and often long-lasting ways. The effects of these processes on developmental risk for pathology are particularly notable. Below we briefly outline the breadth of topics covered herein, as well as how they are integrated and together advance our overall understanding of the neural circuits and processes mediating the stress and fear responses.

The issue begins with an exploration of the effects of stress on neuronal structure and function. This area has led to a number of fascinating insights into the effects of stress and hypothalamic-pituitary-adrenal (HPA) axis function on dendritic retraction and decreased spine density-all cellular measures of decreased cellular functioning and plasticity (McEwen et al, 2016). Following on this, Antoine Besnard and Amar Sahay detail our up-to-date understanding of neurogenesis within the adult brain, particularly within the hippocampus, and how this process may mediate discrimination of discrete memory events. Furthermore, in stress models, this neurogenesis appears to be impaired, in part resulting in the stress- and fear-generalization that occurs with diminished hippocampal neurogenesis (Besnard and Sahay, 2016).

The next chapters focus on an aspect of stress that has been particularly well developed in the last decade-fear regulation and its underlying neural circuity. Michael Fanselow explores the effect of prior stress sensitization on later enhanced fear responses (Perusini et al, 2016), providing a potentially important model helping to provide insight into why a history of prior stress increases the likelihood of PTSD following new trauma exposure. The following chapter builds on these ideas by examining how stress affects contextual fear learning, particularly related to dysregulation of the stress response and fear systems, as occurs in a variety of anxiety disorders (Maren and Holmes, 2016). How fear is modulated by stress is beginning to be understood at a variety of levels, including both neural circuitry and underlying molecular mechanisms. A particularly interesting set of findings over the past few years has revealed how the endogenous cannabinoid system is involved in both extinction of fear and in modulating the stress response, as reviewed in detail at the end of this section (Morena et al, 2016).

Although the amygdala is one of the best understood structures mediating the stress and fear response, a region of the 'extended' amygdala, the bed nucleus of the stria terminalis (BNST) is increasingly being appreciated as a component of anxiety-like responses, perhaps mediating similar survival reflexes as the amygdala, but when the feared cue is anticipated or is at a distance. Rainnie and colleagues provide a thorough review of the electrophysiological underpinnings of the BNST-dependent stress response (Daniel and Rainnie, 2016). This chapter is followed by a review of new findings of BNST function in humans, in particularly how the human BNST modulates anxiety and stress responses using fMRI approaches (Avery et al, 2016).

Differential contexts of stress exposure may be particularly important in understanding how the biology of the brain responds. The next section examines developmental psychopathology, with an initial chapter by Boyce focusing on biology-environment interactions in disease pathogenesis (Boyce, 2016). Nim Tottenham's group has spent years examining the effects of developmental studies of stress on the brain, in particular through an amazing series of studies with children who were raised in situations of social neglect (Callaghan and Tottenham, 2016). Johanna Bick and Charles Nelson then provide further exploration of these topics and mechanisms by examining the developmental consequences of toxic stress exposure (Bick and Nelson, 2016). Finally, within this section, the effects of early life genomic and behavioral programming, and the stress effects of reprogramming in the developing brain are outlined (Chen and Baram, 2016).

In addition to the effects of developmental stress on the brain and behavior, more recent data has begun to outline how intergenerational processes may, in part, be due to epigenetic effects both within the stressed generation, but also in their subsequent generations. Stephanie Bronson and Tracy Bale present a comprehensive review on epigenetic programming of stress pathways via the gametes in chronic stress rodent models (Bronson and Bale, 2016), which is followed by a review focusing on intergenerational mechanisms of transfer of fear and sensory sensitization, in particular an intriguing model of olfactory fear-mediated intergenerational transmission in mice (Klengel et al, 2016). Finally, recent work in humans, in particular through work with offspring of holocaust survivors, has demonstrated that 
intergenerational mechanisms of risk, possibly mediated through epigenetic processes, may also occur in humans (Bowers and Yehuda, 2016).

No review of stress pathways would be complete without updates on recent progress in the HPA stress axis. Muglia and colleagues provide a thorough review of recent work, in particularly using genetic approaches in animal model systems to understand mechanistic regulation of the HPAcortisol system (Arnett et al, 2016). This is followed by a review of gene $\times$ environment $(\mathrm{g} \times \mathrm{e})$ approaches to understanding stress, with a particular focus on the well-worked out example of the FKBP5 pathway, which is now understood to regulate glucocorticoid receptor feedback, with polymorphisms in this gene interacting with childhood trauma to predict differential effects on adult trauma-related sequelae (Zannas et al, 2016).

The topic of $\mathrm{g} \times \mathrm{e}$ interactions is then elaborated with an exciting update and review of $\mathrm{g} \times \mathrm{e}$ models in understanding stress, anxiety, and fear processes as well as their underlying neural circuits in the human brain (Bogdan et al, 2016). A more broad review then follows, covering an up-to-date overview of genetic approaches, from GWAS to epigenetics, in understanding an array of stress-related psychiatric disorders and syndromes (Smoller, 2016). John Greco and Israel Liberzon then provide a nice overview of neural circuit manifestations, based in human imaging studies, of the effects of stress, fear, and fear extinction on circuit processes and activity (Greco and Liberzon, 2016).

Though much work has been done to understand mechanisms, less progress has been made in preventing stress-related disorders, ranging from PTSD to depression. Howlett and Stein review updates on prevention and treatment in this arena and how a newer understanding of stress circuitry and biology may inform novel approaches (Howlett and Stein, 2016). Finally, understanding comorbidity of stress and trauma with other disorders, less typically associated with these circuits, is of critical importance. In particular, understanding how the negative valence systems of the brain interact with the positive valence systems mediating addiction, is both fascinating and important for progress. This area is nicely covered in the final chapter focusing on 20 years of progress in understanding stressinduced reinstatement of drug seeking (Mantsch et al, 2016).

Together, we hope that this collection of outstanding, thorough, and intersecting reviews will help basic scientists and clinicians alike in gaining a greater appreciation for how the processes of stress, fear, and other negative valence systems interact to create psychopathology. Furthermore this work displays a broad array of neuroscience perspectives and approaches, demonstrating with fascinating clarity how the brain, when homeostasis or allostasis is dysregulated through the process of stress (whether external or internal) can lead to emotional dysregulation and behavioral difficulties. We hope that this work is as exciting for you to read as it was for us, and that it catalyzes new ideas at the intersection of neuroscience, psychiatry, and pharmacology. Only through such work will new treatments, interventions, and cures be possible for the enormous morbidity and mortality that derive from stress-related disorders.

\section{FUNDING AND DISCLOSURE}

Dr. Smoller is an unpaid member of the Scientific Advisory Board of PsyBrain, Inc. The other author declared no conflict of interest.

\section{ACKNOWLEDGMENTS}

NIMH grants R01 MH096764 and R01 MH094757 to KJR. NIMH grants R01-MH079799 and K24MH094614 to JWS. Dr. Smoller is the Tepper Family MGH Research Scholar.

\section{REFERENCES}

Arnett MG, Muglia LM, Laryea G, Muglia LJ (2016). Genetic approaches to hypothalamicpituitary-adrenal axis regulation. Neuropsychopharmacology 41: 245-260.

Avery SN, Clauss JA, Blackford JU (2016). The human bnst: functional role in anxiety and addiction. Neuropsychopharmacology 41: 126-141.

Besnard A, Sahay A (2016). Adult hippocampal neurogenesis, fear generalization and stress. Neuropsychopharmacology 41: 24-44.

Bick J, Nelson CA (2016). Early adverse experiences and the developing brain Neuropsychopharmacology 41: 177-196.

Bogdan R, Pagliaccio D, Baranger DAA, Hariri AR (2016). Genetic moderation of stress effects on corticolimbic circuitry. Neuropsychopharmacology 41: 275-296.

Bowers ME, Yehuda R (2016). Intergenerational transmission of stress in humans. Neuropsychopharmacology 41: 232-244.

Boyce WT (2016). Differential susceptibility of the developing brain to contextual adversity and stress. Neuropsychopharmacology 41: 142-162.

Bronson SL, Bale TL (2016). The placenta as a mediator of stress effects on neurodevelopmental reprogramming. Neuropsychopharmacology 41: 207-218.

Callaghan BL, Tottenham N (2016). The Neuro-Environmental Loop of Plasticity: A crossspecies analysis of parental effects on emotion circuitry development following typical and adverse caregiving. Neuropsychopharmacology 41: 163-176.

Chen Y, Baram TZ (2016). Towards understanding how early-life stress re-programs cognitive and emotional brain networks. Neuropsychopharmacology 41: 197-206.

Daniel SE, Rainnie DG (2016). Stress modulation of opposing circuits in the bed nucleus of the stria terminalis. Neuropsychopharmacology 41: 103-125.

Greco JA, Liberzon I (2016). Neuroimaging of fear-associated learning. Neuropsychopharmacology 41: 320-334.

Howlett JR, Stein MB (2016). Prevention of trauma and stressor related disorders: a review. Neuropsychopharmacology 41: 357-369.

Klengel T, Dias BG, Ressler KJ (2016). Models of inter- and transgenerational transmission of risk for psychopathology in mice. Neuropsychopharmacology 41: 219-231.

Mantsch JR, Baker DA, Funk D, Lê AD, Shaham Y (2016). Stress-induced reinstatement of drug seeking: 20 years of progress. Neuropsychopharmacology 41: 335-356.

Maren S, Holmes A (2016). Stress and fear extinction. Neuropsychopharmacology 41: 58-79.

McEwen BS, Nasca C, Gray JD (2016). Stress effects on neuronal structure: hippocampus, amygdala and prefrontal cortex. Neuropsychopharmacology 41: 3-23.

Morena M, Patel S, Bains JS, Hill MN (2016). Neurobiological interactions between stress and the endocannabinoid system. Neuropsychopharmacology 41: 80-102.

Perusini JN, Meyer EM, Long VA, Rau V, Nocera N, Avershal J et al (2016). Induction and expression of fear sensitization caused by acute traumatic stress. Neuropsychopharmacology 41: 45-57.

Smoller JW (2016). The genetics of stress-related disorders: PTSD, depression and anxiety disorders. Neuropsychopharmacology 41: 297-319.

Zannas AS, Wiechmann T, Gassen NC, Binder EB (2016). Gene-stress-epigenetic regulation of FKBP5: clinical and translational implications. Neuropsychopharmacology 41: 261-274.

Kerry J Ressler ${ }^{1}$ and Jordan W Smoller ${ }^{2}$

${ }^{1}$ Division of Depression and Anxiety Disorders, James and Patricia Poitras Chair in Psychiatry, McLean Hospital, Harvard Medical School, Belmont, MA, USA; ${ }^{2}$ Psychiatric and

Neurodevelopmental Genetics Unit, Massachusetts General Hospital, Harvard Medical School, Department of Epidemiology, Harvard T.H. Chan School of Public Health, Stanley Center for

Psychiatric Research, Broad Institute, Boston, MA, USA.

E-mails: kressler@mclean.harvard.edu; jsmoller@hms.harvard.edu 